\title{
Ultrafast double magnetization switching in GdFeCo with two picosecond-delayed femtosecond pump pulses
}

\author{
U. Atxitia ${ }^{1, a)}$ and T. A. Ostler ${ }^{2, b)}$ \\ ${ }^{1}$ Freie Universität Berlin, Fachbereich Physik, Arnimallee 14, 14195 Berlin, Germany \\ ${ }^{2}$ Faculty of Arts, Computing, Engineering and Sciences, Sheffield Hallam University, Howard Street, \\ Sheffield S1 1WB, United Kingdom
}

(Received 12 June 2018; accepted 20 July 2018; published online 8 August 2018)

\begin{abstract}
The recently discovered thermally induced magnetization switching (TIMS) induced by single femtosecond laser pulses in ferrimagnetic GdFeCo alloys proceeds on the picosecond time-scale. The rate at which data can be changed for use of TIMS in technological devices is limited by the processes leading to thermal equilibrium. In the present work, we address the question of whether it is possible to further excite switching via TIMS well before thermal equilibrium between subsystems is reached. In particular, we investigate the conditions for double thermally induced magnetic switching by the application of two shortly delayed laser pulses. These conditions become relevant for potential applications as it sets both a limit to rewrite data and demonstrates the importance of spatial confinement of a heat pulse to bit size, as neighboring bits may be accidentally re-switched for spatially extended pulse spots. To demonstrate this effect, we theoretically study the switching behavior in a prototypical ferrimagnetic GdFeCo alloy as a function of composition. We use computer simulations based on thermal atomistic spin dynamics and demonstrate the possibility of inducing a second switching event well before thermal equilibrium is reached and define the conditions under which it can occur. Our theoretical findings could serve as a guidance for further understanding of TIMS as well as to act as a guide for future applications. Published by AIP Publishing. https://doi.org/10.1063/1.5044272
\end{abstract}

Efficient field-free magnetic switching schemes are drawing great interest as a means of downscaling of devices to the nanoscale. Ultrafast robust magnetic switching in nanodevices is also desirable, where femtosecond laser pulses are the preferred stimulus. The prospectives for femtosecond optonanomagnetism, ${ }^{1}$ as a viable approach for ultrafast information processing technology, were increased after the discovery of the so-called all-optical switching (AOS). ${ }^{2}$

All-optical switching has been found in a broad variety

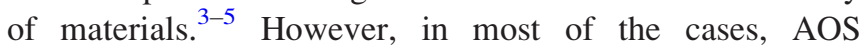
responds to a cumulative effect of multiple pulses with circularly polarised light, and thus is often referred to as helicitydependent all-optical switching (HD-AOS). In comparison, single shot sub-ps magnetic switching has only been achieved in the ferrimagnetic alloy GdFeCo. ${ }^{6-8}$ Importantly, it has been shown that heat alone can deterministically reverse the magnetic polarity, and therefore is often named ultrafast thermally induced magnetization switching (TIMS). This is advantageous over the HD-AOS mechanism as it further simplifies laser spot length reduction ${ }^{9}$ and therefore better integration into current technologies. Moreover, TIMS has been used to induce single shot switching in heterostructures of $\mathrm{GdFeCo} / \mathrm{Co} / \mathrm{Pt}^{10}$ and synthetic $\mathrm{Gd} / \mathrm{Co} / \mathrm{Pt} .{ }^{11}$ Furthermore, beyond the use of laser light, recent studies have demonstrated the possibility to use picosecond electric currents exploiting the thermal origin of TIMS, ${ }^{12}$ opening up new avenues for picosecond spintronics.

How fast can TIMS be repeated is so far unknown. It is assumed that the different subsystems-spin, electron, and

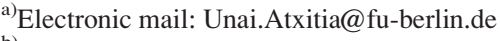

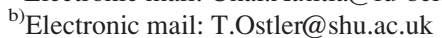

phonon-need to equilibrate and that the spin system returns to its initial state before the process can be repeated. A careful choice of the substrate can reduce the cooling time down to the sub-ns timescale. ${ }^{13}$ Still this fact sets a limit to the repetition rate, which is relevant for applications, ${ }^{14}$ though also has consequences for magnetic recording where neighboring nanograins in a media could be unintentionally reversed if the laser light is not sufficiently confined. It is also crucial for the recently proposed parallel all-optical writing of magnetic domains using spatial and temporal interference of two ultrashort light pulses. ${ }^{15}$ Thus, identifying the conditions under which subsequent switching events can occur, and whether this is limited by the magnetic relaxation (to a given, perhaps elevated temperature), or cooling of the system to ambient (or operating temperature) is of interest both fundamentally and for applications. Further control and manipulation of TIMS by a second pulse on a time scale much shorter than the equilibration time has not been particularly addressed either experimentally nor theoretically. ${ }^{16}$ In this work, we determine the conditions for which a second switching event is possible using two shortly delayed heat pulses by means of computer simulations based on an atomistic spin model.

The energetics of the ferrimagnetic system are described by the classical spin Heisenberg Hamiltonian

$$
\mathcal{H}=-\frac{1}{2} \sum_{\langle i j\rangle} J_{i j} \mathbf{S}_{i} \cdot \mathbf{S}_{j}-\sum_{i} K_{i}\left(S_{i, z}\right)^{2},
$$

where $\langle i j\rangle$ indicates the sum is limited to the nearest neighbor pairs with $\left|\mathbf{S}_{i}\right|=\boldsymbol{\mu}_{i} / \mu_{i}, \boldsymbol{\mu}_{i}$ representing the atomic magnetic moment. The lattice consists of two species of spins which are randomly distributed on a regular, simple cubic 
lattice. The FeCo sublattice has a magnetic moment $\mu_{\mathrm{FeCo}}=1.92 \mu_{\mathrm{B}}$. The Gd sublattice is attributed a moment of $\mu_{\mathrm{Gd}}=7.63 \mu_{\mathrm{B}}$ which takes into account the contribution of the half-filled $4 f$ core electrons $\left(7 \mu_{\mathrm{B}}\right)$ and valence band $5 d$ electrons spin $\left(0.63 \mu_{\mathrm{B}}\right)^{17}$ ( $\mu_{\mathrm{B}}$ is the Bohr magneton). It has been shown that similar results are obtained when the $\mathrm{Gd}$ spin is orbitally resolved. ${ }^{18}$ The values of exchange energy, $J_{i j}$, and magnetic anisotropy $K_{i}$ are taken from Ref. 19 .

The spin dynamics are described by the stochastic Landau-Lifshitz-Gilbert (LLG) equation of motion

$$
\frac{\mathrm{d} \mathbf{S}_{i}}{\mathrm{~d} t}=-\frac{\gamma}{\left(1+\lambda_{i}^{2}\right) \mu_{i}} \mathbf{S}_{i} \times\left[\mathbf{H}_{i, \mathrm{eff}}+\lambda_{i}\left(\mathbf{S}_{i} \times \mathbf{H}_{i, \mathrm{eff}}\right)\right],
$$

where the effective fields are given by $\mathbf{H}_{i, \text { eff }} \equiv-\frac{1}{\mu_{i}} \frac{\partial \mathcal{H}}{\partial \mathbf{s}_{i}}+\zeta_{i}$, where $\mathcal{H}$ is given by Eq. (1). The stochastic fields $\zeta_{i}$ represent the thermal effects with zero mean value $\left\langle\zeta_{i}\right\rangle=0$. The variance of the stochastic process is given by $\left\langle\zeta_{i}^{k}(0) \zeta_{j}^{l}(t)\right\rangle$ $=2 \delta_{i j} \delta_{k l} \delta(t) \mu_{i} \lambda_{i} k_{B} T / \gamma_{i}$, where $i, j$ denote lattice sites and $k$, $l$ are the Cartesian components, $k_{B}$ is the Boltzmann constant, $T$ is the heat-bath temperature, and $\gamma$ is the gyromagnetic ratio. The value of $\lambda_{i}$ determines the rate at which energy and angular momentum are exchanged (into and out of) the thermal bath which we assume (see below) is that of the electron system. $6,19,20$

The dynamics of the electron and lattice energy are modeled by the two-temperature model $(2 \mathrm{TM})^{21}$

$$
\begin{aligned}
C_{\mathrm{e}}\left(T_{\mathrm{e}}\right) \frac{\mathrm{d} T_{\mathrm{e}}}{\mathrm{d} t} & =G_{\mathrm{ep}}\left(T_{\mathrm{e}}-T_{\mathrm{ph}}\right)+P_{1}(t)+P_{2}(t), \\
C_{\mathrm{ph}} \frac{\mathrm{d} T_{\mathrm{ph}}}{\mathrm{d} t} & =-G_{\mathrm{ep}}\left(T_{\mathrm{e}}-T_{\mathrm{ph}}\right)+\frac{T_{\mathrm{ph}}-T_{0}}{\tau_{d}},
\end{aligned}
$$

where $C_{\mathrm{e}}\left(T_{\mathrm{e}}\right)=\gamma_{e} T_{e},{ }^{21}$ with $\gamma_{e}=2.25 \times 10^{2} \mathrm{Jm}^{-3} \mathrm{~K}^{-2}$, $C_{\mathrm{ph}}=3.1 \times 10^{6} \mathrm{Jm}^{-3} \mathrm{~K}^{-1}$, are the electron and phonon specific heats, respectively. The laser pulse energy input is absorbed by the electron system and described by a Gaussian function, $P\left(t_{0}, \tau_{p}\right)=P_{0} \exp \left(\left(-t-t_{0}\right) / \tau_{p}\right)^{2}$, where the first pulse is centered at $t_{0}=0$, and the second at $t_{0}=\Delta t_{\text {pulse }}$ with the laser pulse width, $\tau_{p}=50 \mathrm{fs}$, and $P_{0}$ is the laser input power, see the gray area in Fig. 1. Due to electron-phonon

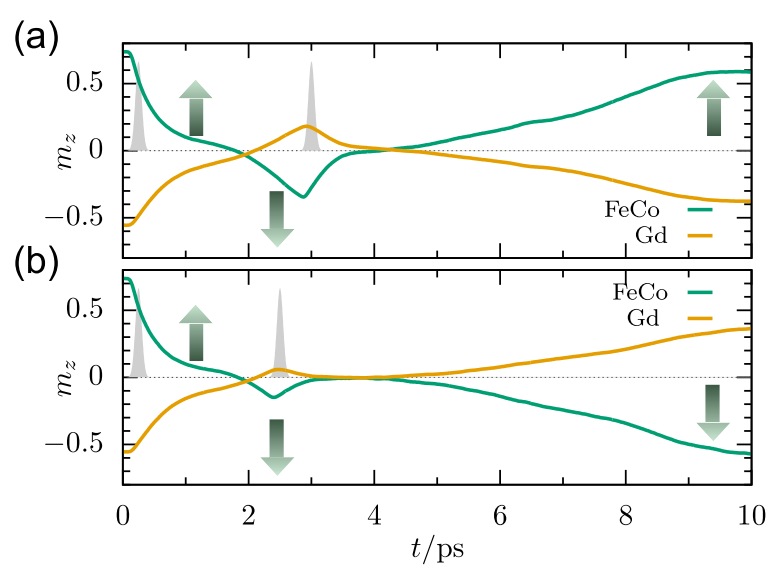

FIG. 1. Element-specific magnetization dynamics of the GdFeCo alloy after the application of two consecutive fs laser pulses (in gray) at certain time delay. (a) For delays larger than $2.5 \mathrm{ps}$, a second switching of the magnetization can be achieved for this composition of GdFeCo, whereas (b) for delays shorter than $2 \mathrm{ps}$, we observe no second switching. interaction, electrons pass energy to the lattice at a rate determined by the electron-phonon coupling, $G_{\mathrm{ep}}=2.5$ $\times 10^{17} \mathrm{Wm}^{-3} \mathrm{~K}^{-1}$. We note here that the value of $G_{\mathrm{ep}}$ gives the minimum required fluence for TIMS, and it is consistent with values found for $\mathrm{Gd}$, but more importantly, relatively close to the recent exquisite estimation by Wilson and co-workers $^{22}$ for $\mathrm{Au} / \mathrm{Gd}_{29}\left(\mathrm{Fe}_{90} \mathrm{Co}_{10}\right)_{71}, \quad G_{\text {ep }}=6 \pm 2.4$ $\times 10^{17} \mathrm{~W} \mathrm{~m}^{-3} \mathrm{~K}^{-1}$. Finally, the phonon and electron systems go back to their initial states by releasing the absorbed energy to the environment on a time scale given by $\tau_{d}$. In the following, we will assume that $\tau_{d}$ is much larger than the time scale of the simulations, e.g., with a glass substrate, so we can neglect the energy diffusion term in the 2TM.

In our simulations, we assume that the fluence of both pulses are the same, which is closer to potential experimental situations. The initial temperature was set at room temperature, $T=300 \mathrm{~K}$. The data sets were averaged over 20 runs with different random number seeds. The system size was $100 \times 100 \times 100$ spins on a simple cubic lattice so the errors in the switching probability are quite low. The fluence, $P_{0}$, was varied to find the minimum value (as a function of composition), $F_{0}^{\min }=P_{0}^{\min } \tau_{p} \sqrt{\pi}$ required for switching with the first laser pulse. To find the minimum energy for TIMS with the first pulse, it is important to avoid overheating by the second laser pulse. The values of fluence used for switching the 30,25 , and $20 \%$ compositions were $1.17 \times 10^{21} \mathrm{~J} / \mathrm{m}^{3} \mathrm{~s}$, $1.35 \times 10^{21} \mathrm{~J} / \mathrm{m}^{3} \mathrm{~s}$, and $1.85 \times 10^{21} \mathrm{~J} / \mathrm{m}^{3} \mathrm{~s}$, respectively. A minimum appears at Gd concentrations around $30 \%$ which is in agreement with our recent predictions. ${ }^{19,23}$ We start our investigation with this concentration.

Figure 1(a) shows an example of the element-specific spin dynamics during two-pulse switching. After the application of the first laser pulse, with sufficient energy to induce TIMS, the Gd and FeCo magnetization reduces substantially in the first two picoseconds. Importantly, the rate of demagnetization is different for each sublattice, one of the key conditions for TIMS, and in agreement with experiments. ${ }^{24}$ Following demagnetization, the FeCo sublattice switches polarity followed by $\mathrm{Gd}$ magnetization. After the first switching event, the magnetization of both sublattices starts to recover. For the second laser pulse, we find that for delays longer than $t=2.75 \mathrm{ps}$, a second TIMS event occurs. Similar to the first laser pulse, after the second laser pulse, FeCo and Gd demagnetize and switch. By exploring different time lapses, we find that for a time delay of $t=2.25 \mathrm{ps}$, a second TIMS is not reproduced, see Fig. 1(b).

The question now is how much can the time-delay be reduced whilst still observing a second TIMS event and how does it depend on the material parameters? Already, for single pulse TIMS, we have shown in previous studies that the 2TM parameter values, e.g., electron-phonon coupling, could determine the minimum value for the laser fluence. In this work, we fix the 2TM parameters to those that closer reproduce the experimental observations. Additionally, material parameters can also potentially influence the switching characteristics for both one and two pulses. In the present study, we have chosen to fix most of the magnetic parameters, and we focus on the effect of the Gd concentration. This is relevant since the minimum laser energy to induce TIMS is closely related to the temperature dependence of the total 
magnetization, ${ }^{19,25}$ which in turn strongly depends on the Gd concentration (see Fig. 2). In particular, we have shown previously that this minimum laser energy scales with the energy gap between the two ferrimagnetic precession modes, i.e., $\hbar \Delta \omega \sim J_{\mathrm{RT}}\left(M_{\mathrm{FeCo}}\left(T_{0}\right)-M_{\mathrm{Gd}}\left(T_{0}\right)\right)$. where $M_{\mathrm{FeCo}(\mathrm{Gd})}\left(T_{0}\right)$ are the individual sublattice magnetizations at temperature, $T_{0}$. Therefore, TIMS needs less energy where the energy gap is minimum, namely close to the so-called compensation temperature $T_{M}$, defined as $M_{\mathrm{FeCo}}\left(T_{M}\right)=M_{\mathrm{Gd}}\left(T_{M}\right)$. We showed in Ref. 19 that this minimum energy gap can be slightly modified by the presence of clustering. In addition to the "close to $T_{M}$ " rule for TIMS, we have previously found a second rule; non-equivalent heating efficiency of the two sublattices, reflected in the distinct demagnetization dynamics. ${ }^{25}$ Information about the non-equivalence criteria can be directly extracted from experimentally measurable quantities, such as the rate of change, $\partial M / \partial T$, of the net equilibrium magnetization $M(T)=M_{\mathrm{Gd}}(T)-M_{\mathrm{FeCo}}(T)$ (Fig. 2). For temperature regions where $\partial M / \partial T \lessgtr 0$ the heating efficiency is different for each sublattice, $\partial M_{\mathrm{Gd}} / \partial T \lessgtr \partial M_{\mathrm{FeCo}} /$ $\partial T$ effectively means that the Gd sublattice reacts slower/ faster than the $\mathrm{FeCo}$ sublattice to a heat pulse. When $\partial M_{\text {net }} / \partial T \simeq 0$ (gray area Fig. 2), both magnetic sublattices are equivalent and TIMS does not occur (see the gray area in Fig. 2). This phenomenology has proven very helpful in the prediction of the ultrafast magnetization dynamics in GdFeCo. ${ }^{24,25}$

The question here is whether or not this phenomenology can be applied in a non-equilibrium situation, e.g., right after the first TIMS event when basically spin, electron, and phonon subsystems are barely in equilibrium with each other. In the following, however, we test the validity of the rule $\partial M / \partial T \lessgtr 0$, where $M$ is time-dependent, to predict conditions for double switching, and extend the second rule for TIMS to non-equilibrium situations. For $x=30 \%$, in Fig. 1(a) we observe that the magnetization is completely reduced after the first switching, this state can be fairly considered to be above $T_{c}$. Within our framework, the second rule states that in order to complete a second switching, the magnetic state needs to be at a temperature below the corresponding gray area in Fig. 2, where $\partial M / \partial T<0$. This crossing from

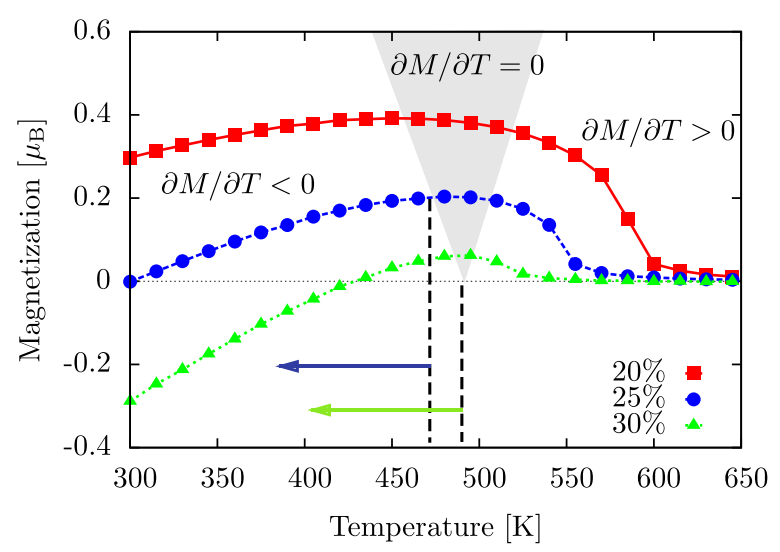

FIG. 2. Equilibrium magnetization of $\mathrm{Gd}_{x}(\mathrm{FeCo})_{1-x}$ alloys with $x=20,25$, and $30 \%$ gained from the atomistic spin model. The grey area represents the temperature region where the element specific relaxation rate is similar and TIMS is unlikely to happen. The arrows point out the temperature region for which TIMS is possible.
$T_{c}$ to $\partial M / \partial T<0$ takes some time which is reflected in the probability to switch as a function of the pulse delay shown in Fig. 3. For $x=30 \%$, a sharp transition from 0 to 1 is observed. For a Gd concentration of $x=25 \%$, the system needs to cool down for a longer time for a second switching since the temperature area where $\partial M / \partial T \approx 0$ is larger than for $x=30 \%$. An additional effect of a larger plateau $\partial M_{\text {net }} / \partial T \simeq 0$ (Fig. 2) for $x=25 \%$ than for $x=30 \%$, translates into a regime of time delays between pulses where switching becomes stochastic, with probabilities neither 0 nor 1, as can be seen in Fig. 3. This can be clearly seen when the Gd concentration is reduced to $x=20 \%$ where the plateau spans a large temperature range, where the probability of double-switching transitions from no-double-switching to completely stochastic, with probability 0.5 . The reason behind this is that the minimum fluence necessary for TIMS for $x=20 \%$ is higher than for $x=30 \%$ and $25 \% .{ }^{19}$ As a consequence, the electron temperature after the second laser pulse remains around $\sim 420 \mathrm{~K}$, where $\partial M / \partial T \approx 0$. In order to reproduce TIMS with a second laser pulse for $x=20 \%$, the electron temperature would need to be further reduced to the temperature region, where $\partial M / \partial T>0$. This cooling process is defined by heat diffusion to other subsystems, such as the substrate or the environment, which are relatively slow processes. We should point out that different substrates, e.g., metallic rather than glass, could significantly reduce the diffusion time and a second switching event may be possible. Thus, although TIMS is permitted for a broad range of $\mathrm{Gd}$ concentrations for the case of a single laser pulse, the double switching is restricted to a narrower concentration region $(x=25 \%-30 \%)$.

To summarize, we have demonstrated through theoretical modeling the possibility of double-ultrafast thermally induced magnetization switching in different compositions of $\mathrm{Gd}_{x} \mathrm{FeCo}_{1-x}$. We find a strong compositional dependence on the ability to induce a second switching event within a few picoseconds, as well as for the minimum time-delay between the two pulses. We explain this time-delay by building on our previous studies ${ }^{19}$ that discuss the compositional

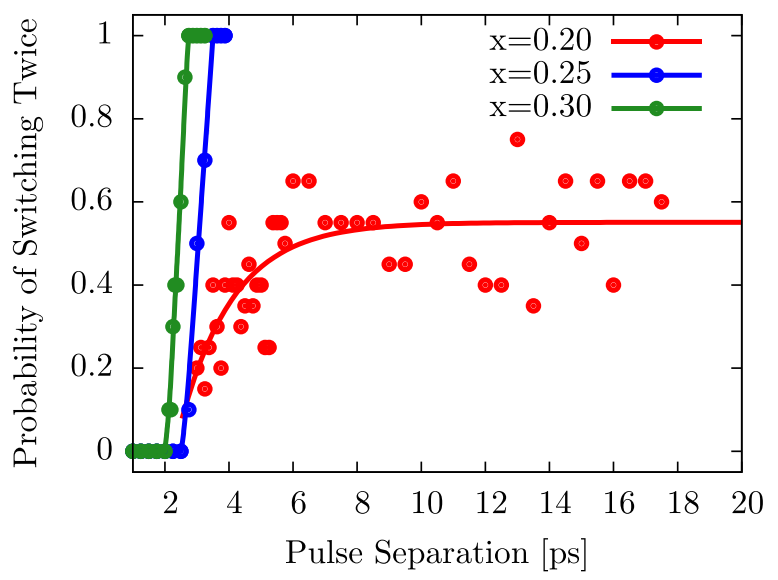

FIG. 3. Probability of inducing two switching events as a function of pulse separation for two compositions of GdFeCo. The green points are for $30 \%$ $\mathrm{Gd}$, the blue points are for $25 \%$, and the red points are for $20 \%$. Non-integer probability of switching twice is related to the crossing of the gray area $(\partial M / \partial T=0)$ of the magnetic state when cooling down after the first TIMS event. 
dependence of the energy required to induce a single switching event and an empirical model based on the rate of change of the equilibrium magnetization. Furthermore, our results open the possibility to induce further switching events. Although a number of parameters would have to be further optimized, such as fluence, Gd concentration, heat diffusion, electro-phonon coupling, and separation between pulses. Whilst quantitatively our results are restricted to $\mathrm{GdFeCo}$, this effect should not be restricted to this material alone. Several studies have recently investigated the possibility of switching in other types of RE-TM alloys, ${ }^{26,27}$ as well as synthetic ferrimagnetic structures. ${ }^{28,29}$ We hope that our findings will invoke new experimental measurements on the possibility of inducing multiple rapid switching events, which could potentially be used for magnetic writing schemes.

Financial support by the Deutsche Forschungsgemeinschaft through SFB/TRR 227 "Ultrafast Spin Dynamics," Project A08, is gratefully acknowledged.

${ }^{1}$ A. Kirilyuk, A. V. Kimel, and T. Rasing, Rev. Mod. Phys. 82, 2731 (2010). ${ }^{2}$ C. Stanciu, F. Hansteen, A. Kimel, A. Kirilyuk, A. Tsukamoto, A. Itoh, and T. Rasing, Phys. Rev. Lett. 99, 047601 (2007).

${ }^{3}$ S. Mangin, M. Gottwald, C.-H. Lambert, D. Steil, V. Uhlír, L. Pang, M. Hehn, S. Alebrand, M. Cinchetti, G. Malinowski et al., Nat. Mater. 13, 286 (2014).

${ }^{4}$ C.-H. Lambert, S. Mangin, B. S. D. C. S. Varaprasad, Y. K. Takahashi, M. Hehn, M. Cinchetti, G. Malinowski, K. Hono, Y. Fainman, M. Aeschlimann et al., Science (New York, N. Y.) 345, 1337 (2014).

${ }^{5}$ R. John, M. Berritta, D. Hinzke, C. Müller, T. Santos, H. Ulrichs, P. Nieves, J. Walowski, R. Mondal, O. Chubykalo-Fesenko et al., Sci. Rep. 7, 4114 (2017).

${ }^{6}$ T. A. Ostler, J. Barker, R. F. L. Evans, R. W. Chantrell, U. Atxitia, O. Chubykalo-Fesenko, S. El Moussaoui, L. Le Guyader, E. Mengotti, L. J. Heyderman et al., Nat. Commun. 3, 666 (2012).

${ }^{7}$ L. Le Guyader, S. El Moussaoui, M. Buzzi, R. V. Chopdekar, L. J. Heyderman, A. Tsukamoto, A. Itoh, A. Kirilyuk, T. Rasing, A. V. Kimel et al., Appl. Phys. Lett. 101, 022410 (2012).
${ }^{8}$ A. R. Khorsand, M. Savoini, A. Kirilyuk, A. V. Kimel, A. Tsukamoto, A. Itoh, and T. Rasing, Phys. Rev. Lett. 110, 107205 (2013).

${ }^{9}$ B. Koene, M. Savoini, A. V. Kimel, A. Kirilyuk, and T. Rasing, Appl. Phys. Lett. 101, 013115 (2012).

${ }^{10}$ J. Gorchon, C.-H. Lambert, Y. Yang, A. Pattabi, R. B. Wilson, S. Salahuddin, and J. Bokor, Appl. Phys. Lett. 111, 042401 (2017).

${ }^{11}$ M. L. M. Lalieu, M. J. G. Peeters, S. R. R. Haenen, R. Lavrijsen, and B. Koopmans, Phys. Rev. B 96, 220411 (2017).

${ }^{12}$ Y. Yang, R. B. Wilson, J. Gorchon, C.-H. Lambert, S. Salahuddin, and J. Bokor, Sci. Adv. 3, e1603117 (2017).

${ }^{13}$ A. Hassdenteufel, C. Schubert, B. Hebler, H. Schultheiss, J. Fassbender, M. Albrecht, and R. Bratschitsch, Opt. Express 22, 10017 (2014).

${ }^{14}$ Z. A. Azim, X. Fong, T. Ostler, R. Chantrell, and K. Roy, IEEE Electron Device Lett. 35, 1317 (2014), 1410.2887.

${ }^{15}$ C. S. Davies, J. Janušonis, A. V. Kimel, A. Kirilyuk, A. Tsukamoto, T. Rasing, and R. I. Tobey, J. Appl. Phys. 123, 213904 (2018).

${ }^{16}$ S. Alebrand, A. Hassdenteufel, D. Steil, M. Cinchetti, and M. Aeschlimann, Phys. Rev. B 85, 092401 (2012).

${ }^{17}$ J. Jensen, Rare Earth Magnetism: Structures and Excitations (Clarendon Press, Oxford, 1991).

${ }^{18}$ S. Wienholdt, D. Hinzke, K. Carva, P. M. Oppeneer, and U. Nowak, Phys. Rev. B 88, 020406 (2013).

${ }^{19}$ J. Barker, U. Atxitia, T. A. Ostler, O. Hovorka, O. Chubykalo-Fesenko, and R. W. Chantrell, Sci. Rep. 3, 3262 (2013).

${ }^{20}$ N. Kazantseva, D. Hinzke, U. Nowak, R. Chantrell, U. Atxitia, and O. Chubykalo-Fesenko, Phys. Rev. B 77, 184428 (2008).

${ }^{21}$ J. Chen, D. Tzou, and J. Beraun, Int. J. Heat Mass Transfer 49, 307 (2006).

${ }^{22}$ R. B. Wilson, C.-H. Lambert, J. Gorchon, Y. Yang, S. Salahuddin, and J. Bokor, preprint arXiv:1609.00648 (2016).

${ }^{23}$ U. Atxitia, T. A. Ostler, R. W. Chantrell, and O. Chubykalo-Fesenko, Appl. Phys. Lett. 107, 192402 (2015).

${ }^{24}$ I. Radu, K. Vahaplar, C. Stamm, T. Kachel, N. Pontius, H. A. Dürr, T. A. Ostler, J. Barker, R. F. L. Evans, R. W. Chantrell et al., Nature 472, 205 (2011).

${ }^{25}$ U. Atxitia, J. Barker, R. W. Chantrell, and O. Chubykalo-Fesenko, Phys. Rev. B 89, 224421 (2014).

${ }^{26}$ R. Moreno, T. A. Ostler, R. W. Chantrell, and O. Chubykalo-Fesenko, Phys. Rev. B 96, 014409 (2017).

${ }^{27}$ X. Lu, X. Zou, D. Hinzke, T. Liu, Y. Wang, T. Cheng, J. Wu, T. A. Ostler, J. Cai, U. Nowak et al., Appl. Phys. Lett. 113, 032405 (2018).

${ }^{28}$ R. F. L. Evans, T. A. Ostler, R. W. Chantrell, I. Radu, and T. Rasing, Appl. Phys. Lett. 104, 082410 (2014).

${ }^{29}$ C. Xu, T. A. Ostler, and R. W. Chantrell, Phys. Rev. B-Condens. Matter Mater. Phys. 93, 054302 (2016). 